\title{
Produção do cuidado em saúde com foco na Clínica Ampliada: um debate necessário na formação em Odontologia
}

\author{
Vinícius Antério Graff*; Ramona Fernanda Ceriotti Toassi** \\ * Cirurgião-dentista do Serviço de Saúde Comunitária, Grupo \\ Hospitalar Conceição/RS \\ ** Professora do Programa de Pós-graduação em Ensino na \\ Saúde da Faculdade de Medicina e da Faculdade de \\ Odontologia, Universidade Federal do Rio Grande do Sul
}

Recebido em 31/08/2017. Aprovado em 04/12/2017.

\begin{abstract}
RESUMO
Este artigo discute a produção do cuidado em saúde, com foco na clínica ampliada na formação em Odontologia. Está organizado em quatro temas principais de análise que são a produção do cuidado em saúde; humanização na saúde e Clínica Ampliada; inovações na produção do cuidado nas práticas em saúde bucal e Abordagem Centrada na Pessoa. Considera que a prática de cuidado em saúde baseada em tecnologias leves-relacionais deve orientar a formação de cirurgiões-dentistas, caracterizando uma formação pela escuta e produção de diálogo, vínculo e subjetividades, de compartilhamento e construção de planos terapêuticos, com uma visão integral e humanizada da atenção à saúde bucal e que incorpore no seu saber-fazer as diretrizes do Sistema Único de Saúde (SUS). Apresenta a Abordagem Centrada na Pessoa como uma das formas de atuação no cuidado em saúde voltado ao usuário e suas potencialidades, e não para a doença. Os currículos da graduação dos cursos de Odontologia devem incluir bases teóricas e oportunidades de vivências e simulações clínicas com foco nas tecnologias leves-relacionais. Tais perspectivas teóricas não devem se restringir à graduação, mas sim, seguir como fundamentais na educação dos profissionais da saúde na formação de pós-graduação, na formação de professores universitários e na educação permanente dos trabalhadores do SUS.
\end{abstract}

Descritores: Educação em Odontologia. Saúde Bucal. Cuidados Integrais de Saúde.

\section{INTRODUÇÃO}

No Brasil, a inclusão das Equipes de Saúde Bucal (ESB) na Estratégia Saúde da Família $(E S F)^{1}$ e o estabelecimento de diretrizes da Política Nacional de Saúde Bucal ${ }^{2}$, possibilitaram a reestruturação e reorganização da saúde bucal no Sistema Único de Saúde (SUS), tendo no conceito de cuidado seu eixo principal. Foram avanços construídos a partir de um processo de discussões que envolveram coordenadores estaduais de saúde bucal e que se fundamentaram nas proposições oriundas de 
deliberações das Conferências Nacionais de Saúde e da I e II Conferências Nacionais de Saúde Bucal e de congressos e encontros de Odontologia e de Saúde Coletiva ${ }^{2}$.

Alia-se a esse contexto de mudanças na saúde, a implementação pelo Conselho Nacional de Educação, em 2002, de Diretrizes Curriculares Nacionais (DCN) para os cursos de graduação em Odontologia ${ }^{3}$, indicando um ensino mais integrado às necessidades do país e aos serviços públicos de saúde. Outras estratégias que impulsionaram mudanças curriculares na graduação, reforçando a integração ensinoserviço, foram o Programa Nacional de Reorientação da Formação Profissional em Saúde - PRÓ-SAÚDE ${ }^{4}$, o Programa de Educação pelo Trabalho para a Saúde - PET-Saúde ${ }^{5}$ e as residências multiprofissionais em saúde ${ }^{6}$.

Houve a qualificação, assim, tanto do ensino, quanto das ações em saúde bucal, com uma prática mais integral, porém, com avanços menores no que se refere à produção de tecnologias de cuidado em ambiente clínico ${ }^{7}$. Não são incomuns episódios de descontentamento na relação entre usuários e dentistas ${ }^{8}$. Os relatos vão desde 'ruídos' na comunicação, como o excessivo uso de termos técnicos, até a frieza do profissional diante de determinadas situações de vida do usuário, com ausência de acolhimento, escuta e diálogo ${ }^{8}$. Cabe considerar que são inabilidades que não se restringem aos profissionais que atuam no SUS.

Compreender a importância da qualidade da relação estabelecida entre usuário e profissional para as ações de atenção à saúde, faz com que isso passe a fazer parte dos objetivos dos próprios profissionais da saúde ${ }^{9}$. Para o alcance de um profissional preparado para dar respostas às necessidades de saúde da população, compreendendo-a na sua subjetividade e individualidade ${ }^{3}$ e buscando a integralidade do cuidado, e fundamental pensar possibilidades para a clínica odontológica que envolvam as subjetividades como uma das dimensões do modo de produção do cuidado em saúde e o uso das tecnologias leves-relacionais é fundamental.

Entendendo este como um debate necessário no processo de formação em odontologia, o presente artigo constitui-se como ensaio $^{10}$ e pretende discutir a produção do cuidado em saúde, com foco na clínica ampliada, à luz dos conceitos de tecnologias levesrelacionais do cuidado, da Política Nacional de Humanização e da Abordagem Centrada na Pessoa. Está organizado em quatro temas principais de análise, que são a produção do cuidado em saúde; humanização na saúde e clínica ampliada; inovações na produção do cuidado nas práticas em saúde bucal e Abordagem Centrada na Pessoa.

\section{PRODUÇÃO DO CUIDADO EM SAÚDE}

Pode-se dizer que o cuidado em saúde é uma relação que se estabelece entre pessoas, e que busca o alívio de um sofrimento ou o alcance de um bem-estar ${ }^{11}$. Para além do atendimento das necessidades sentidas, do uso do saber profissional e das tecnologias necessárias, o cuidado inclui o envolvimento e o relacionamento entre as partes, compreendendo acolhimento com escuta do sujeito, abrindo espaço para as subjetividades do outro, considerando seu contexto sociocultural e histórias de vida ${ }^{12,13}$.

A subjetividade é pelas crenças e valores de cada indivíduo, com suas experiências e histórias de vida. "Pode mudar ao longo do tempo e determina a forma com que $o$ trabalhador analisa e intervém sobre o mundo do trabalho em saúde ${ }^{14,15}$.

O modo de produção do cuidado de cada profissional de saúde está relacionado com suas subjetividades na execução do ato de trabalho. Esse trabalho vivo, em ato, marca o momento 
exato em que o cuidado é executado. Segundo Merhy, em $2000^{15}$, o trabalho em saúde se efetiva na interseção dos desejos e que se estabelece no encontro entre usuário e profissional, ou seja, temos $\mathrm{o}$ ato do profissional de saúde e a necessidade do usuário de restituir a autonomia em sua vida. Esse espaço 'intercessor' é o lugar da relação e de produção comum entre os sujeitos, onde ambos são afetados ${ }^{15,16}$.

O cirurgião-dentista não pode ignorar o estado emocional da pessoa que está recebendo o cuidado e, nesse sentido, aumentar o vínculo afetivo é vital para o bom andamento do tratamento odontológico ${ }^{17}$. A produção de subjetividade é tão singular quanto possam ser as suas vivências. Há necessidade de incorporar e exercitar o uso das tecnologias de relação no processo de formação em Odontologia a fim de promover não só prevenção e autocuidado, mas principalmente a autonomia dos sujeitos. Evidentemente, o trabalhador em saúde não é o único sujeito que cuida e tampouco que tem vontades e desejos, transformando o espaço da clínica num espaço de negociação, uma vez que a imposição não é efetiva ${ }^{18}$.

Quando centralizado exclusivamente em protocolos técnicos de assistência, o trabalho em saúde bucal faz com que os profissionais interajam pouco com as pessoas, desconhecendo suas histórias de vida. Há um desvio do foco da ação cuidadora quando esta se torna refém de um mecanicismo terapêutico, que em detrimento da escuta qualificada, passa a orientar a clínica pela doença. Como contraponto, pode-se afirmar que um processo de cuidado permeável para profissional e usuário, e assim haver o encontro de subjetividades ${ }^{19}$.

Merhy $^{20}$ aposta nas tecnologias leves tecnologia de relações -, para a ampliação das possibilidades terapêuticas e no redirecionamento do modelo de saúde, como atitudes para o envolvimento das subjetividades dos diversos atores que participam do processo de cuidado. Essa tecnologia pode ser transposta para o processo de formação em Odontologia uma vez que a boca não se encontra isolada, mas sim dentro de um corpo, que é resultado do biológico, do psíquico e do afetivo ${ }^{21}$. Respeitar o ritmo do usuário, convocando-o, porém, ao tratamento que demanda e que não se permite realizar; considerar a presença de questões subjetivas que impedem a transformação do desejo do tratamento dentário em ato; e entender a importância dessas questões pode promover a confiança e desencadear a ação de permitir-se ao tratamento.

Essas são situações onde o vínculo é estabelecido, na medida em que o profissional se mostra acessível e, a partir desse momento, há o encontro terapêutico entre usuário e trabalhador da saúde. Em uma consulta odontológica, está em questão não só o procedimento, mas a relação que se estabelece com a pessoa. O profissional precisa estar atento aos sinais que não são sempre falados, como por exemplo, o choro e o medo. A escuta significa, num primeiro momento, acolher toda queixa ou relato do usuário mesmo quando possa parecer não interessar diretamente para o diagnóstico e tratamento ${ }^{22}$.

"Para que se realize uma clínica adequada é preciso saber, além do que o sujeito apresenta de igual, o que ele apresenta de diferente, de singular, inclusive, um conjunto de sinais e sintomas que somente nele se expressam de determinado modo. Com isso, abrem-se inúmeras possibilidades de intervenção, e é possível propor tratamentos muito melhores com a participação das pessoas envolvidas" 22 .

Nesse contexto, o entendimento do cuidado baseado em tecnologias leves deveria orientar a formação/atuação profissional. E então haveria a constituição de um profissional com conhecimentos e também acolhedor, confiável, responsável e capaz de estabelecer uma relação 
de vínculo e aceitação, portanto, capaz do uso e domínio dessas tecnologias relacionais na sua prática $^{23}$. No campo da educação superior, tornase um desafio a incorporação de condutas profissionais 'relacionais' na clínica pelos estudantes. Como esse cuidado vem sendo trabalhado, tendo em vista a operacionalização das Diretrizes Curriculares? $\mathrm{O}$ conceito de tecnologia leve é conhecido pelos professores? Qual o espaço no currículo da graduação para as tecnologias 'leves-duras' e 'duras' da profissão odontológica e para aquelas voltadas para dimensões do cuidado que envolvam as tecnologias leves/relacionais?

Os cursos de graduação na área da saúde, no Brasil, reformularam seus currículos e projetos pedagógicos, agregando inovações curriculares e desenvolvendo experiências em um contexto de currículo integrado, de ações interdisciplinares ${ }^{24}$, entretanto, o desafio é mapear e entender qual o papel das práticas de ensino no desenvolvimento de tecnologias leves/relacionais para o cuidado em saúde bucal.

Modificar a lógica de organização do processo ensino-aprendizagem na Educação Superior nos cursos da saúde pode se constituir um processo árduo. Mitre et al. ${ }^{25}$ destacam que, por um lado, deve-se buscar a autonomia do aluno, colocando-o no lugar de protagonismo em relação a busca de conhecimentos no processo de inovação curricular, cabendo ao docente o papel de facilitador do processo de ensino aprendizagem. Estudos sobre propostas de mudanças nos Projetos Pedagógicos, currículos inovadores e integrados no Brasil são bastante encontrados, porém, pesquisas que tratem da necessidade de formação docente voltada para essa lógica, não são tão comuns ${ }^{24}$.

Entende-se que mudanças no ensino são graduais, alterações nos currículos e práticas inovadoras devem ser constantemente avaliadas e modificadas conforme a necessidade. A formação docente também precisa acompanhar tais processos de mudança. Considerar a dimensão do cuidado em saúde baseado em tecnologias leves é uma possibilidade para profissionais formados com uma visão mais integral e humanizada e que incorporem no seu saber-fazer as diretrizes do SUS.

\section{HUMANIZAÇÃO NA SAÚDE E CLÍNICA AMPLIADA}

A Política Nacional de Humanização (PNH) ${ }^{26}$ foi implementada também no ano de 2004, como a PNSB, trazendo a prerrogativa da valorização das dimensões subjetiva e social em todas as práticas de atenção e gestão ${ }^{27}$ e avançando em relação a questões éticas da relação usuário-trabalhador e de respeito ao usuário do SUS $^{28}$.

Atuando de forma transversal às demais políticas, foram definidas diretrizes gerais para implementação da PNH, sendo que uma delas diz respeito ao conceito de clínica ampliada. $\mathrm{O}$ termo 'ampliada' foi empregado para dimensionar a atuação clínica para além das abordagens convencionais e protocolos de atendimento e a valorização/ compreensão do sujeito no seu contexto familiar, social e de mundo ${ }^{27,29}$. Considera o estímulo a diferentes práticas terapêuticas e corresponsabilidade de gestores, trabalhadores e usuários no processo de produção de saúde ${ }^{26}$. Tal perspectiva pode ser empregada sem abrir mão dos recursos biomédicos de intervenção, mas reconhecendo sua insuficiência para dar conta da complexidade das situações ${ }^{30}$.

Todo profissional que realiza atendimentos/cuidados de pessoas realiza clínica. Cada núcleo profissional realiza uma modalidade de clínica, dentre as quais a Odontologia. Clínica Ampliada é a redefinição (ampliada) do objeto, do objetivo e dos meios de trabalho da assistência individual, familiar ou a grupos. Considera que o 'objeto de trabalho' da assistência à saúde é uma 
pessoa, ou um grupo, ou uma família, ou um coletivo institucional, com doença ou risco de adoecer $^{31}$.

Dessa forma, ocorre a ampliação dos recursos de trabalho, deslocando o foco da intervenção dos medicamentos e procedimentos para a escuta e o diálogo. A Clínica Ampliada é caracterizada pelo olhar singular ao Outro, pela superação da fragmentação do cuidado, responsabilização e vínculo com usuários dos serviços de saúde, ampliação do grau de autonomia do sujeito, valorização da intersetorialidade e o reconhecimento dos limites da medicina e das tecnologias utilizadas. Ainda, utiliza a identificação de fatores de risco e proteção e a valorização do papel terapêutico da escuta, da palavra e da educação em saúde ${ }^{22,30-34}$.

Assim, a clínica ampliada pode ser considerada uma proposta de orientação para o trabalho e para o ensino na área da saúde ${ }^{33}$ e na Odontologia tem potencial para melhorar a visão do cuidado integral das pessoas na clínica odontológica, fortalecendo a formação acadêmica e o cuidado prestado à comunidade ${ }^{27}$.

\section{INOVAÇÕES NA PRODUÇÃO DO CUIDADO NAS PRÁTICAS EM SAÚDE BUCAL}

A ampliação da clínica de saúde bucal parte do pressuposto que, historicamente, o modelo de atenção adotado manteve a saúde bucal isolada em relação às práticas integrais de saúde. Dessa forma, justificam-se experiências de uma clínica ampliada de saúde bucal na perspectiva da integralidade, com a utilização de novas tecnologias de cuidado, baseadas principalmente nas tecnologias leves. É a possibilidade da inovação, mudanças ou novos arranjos nos processos de trabalho, que propiciam o aumento da eficácia, redução de custos, danos ou desperdícios e aumento do bemestar dos usuários ${ }^{35}$.
"Novas abordagens para o cuidado, na direção do sujeito e não mais da doença, mesmo que inicialmente causem estranhamento, acabam por produzir alguns efeitos para uma desnaturalização do cotidiano dos serviços de saúde bucal no SUS" ${ }^{36}$.

Estudo de Barros e Botazzo ${ }^{7}$ mostra uma intervenção em saúde bucal realizada a partir dos pressupostos de atendimento do problema bucal mais relevante ao usuário, 'descentramento dentário', constituição de caso clínico por meio da anamnese e integração com a equipe da Unidade de Saúde. O atendimento era realizado em mesa para consulta, e o exame clínico bucal e o levantamento da história do usuário eram registrados em prontuário único, sem o uso de odontograma. Os resultados apontaram para a importância das habilidades de comunicação e para um vínculo caracterizado pelo diálogo, pela tomada de responsabilidades pelos sujeitos e pela resolução das necessidades do usuário.

Botazzo et al. ${ }^{35}$ relataram, ainda, duas experiências no processo de cuidado na clínica odontológica. A primeira é a anamnese coletiva, atividade em grupo onde a parte inicial das informações da ficha odontológica é preenchida e discutida entre os participantes, propiciando o vínculo usuário-profissional; o exame físico e os registros em prontuário único eram realizados após a atividade com o grupo. A segunda trata-se da consulta em saúde bucal, onde são realizados o acolhimento inicial, a anamnese e exame físico; solicitações de exames complementares e prescrições. Essa modalidade de consulta pode ser realizada em salas de atendimento de uso comum a outros profissionais, dentro da Unidade de Saúde, não necessitando da cadeira odontológica, com o objetivo de escutar o usuário e constituir o caso clínico. Em ambas as experiências, a anamnese não se restringia à que é usualmente praticada. Relacionava outros eventos observáveis na pessoa, tais como 
possíveis manifestações sistêmicas da doença bucal ou vice-versa, e considerava os aspectos psicossociais e de contexto de vida ${ }^{35,37}$.

\section{ABORDAGEM CENTRADA NA PESSOA}

Uma das formas de atuação no cuidado em saúde voltada ao usuário e suas potencialidades, e não à doença, que trata das relações interpessoais é a Abordagem Centrada na Pessoa (ACP). Segundo a ACP, a personalidade humana tem uma tendência para o crescimento e para a saúde, por meio de atitudes e recursos interventivos que permitem o resgate do potencial realizador existente nos seres humanos - a 'Tendência Atualizante' - e reconhecido como o pilar dessa teoria ${ }^{38}$. As atitudes que facilitam essa 'Tendência Atualizante' são a consideração empática, a consideração positiva incondicional e a autenticidade (ou congruência). A consideração empática implica na consideração do outro, de seu mundo subjetivo próprio, de seu campo fenomenológico. Segundo Rogers e Kinget (1977) ${ }^{39}$, é a capacidade de se imergir no mundo subjetivo do outro e de participar da sua experiência, na extensão em que a comunicação verbal ou não-verbal o permite. É a capacidade de se colocar verdadeiramente no lugar do outro, de ver o mundo como ele o vê $\hat{e}^{39}$. A consideração positiva incondicional consiste numa postura ou atitude de consideração irrestrita, numa atitude de abstenção de julgamentos, o que implica na não oposição a qualquer elemento expresso, verbal ou não verbal, direta ou indiretamente pela pessoa. Já a autenticidade significa que o indivíduo seja aquilo que realmente é, no momento da relação. Inclui qualquer forma de ser, enquanto esta mesma seja verdadeira. É o oposto de apresentar uma 'fachada', quer ele tenha ou não conhecimento disso. A união dessas condições em qualquer relacionamento interpessoal seria necessária e suficiente para o desenvolvimento da pessoa ${ }^{39}$.

O campo de aplicação da ACP é bastante amplo, com repercussões nas áreas da psicologia, medicina, enfermagem e terapia ocupacional. Sua utilização na odontologia vem sendo estudada na literatura internacional, tanto em estudos de revisão sistemática ${ }^{40}$, quanto nos que propõem a elaboração de modelos teóricos para sua aplicação ${ }^{41}$. Os resultados desses estudos apontaram para uma escassez de evidências em relação à utilização e sistematização da ACP na Odontologia, bem como para um modelo teórico preliminar que agregasse às dimensões funcionais, dimensões relacionais, como vínculo, valores, empoderamento, atitude e comunicação.

Entende-se, desse modo, que todo trabalho que envolva relações humanas, pode adotar a ACP como forma de lidar com o outro, valorizando a potencialidade terapêutica da relação.

\section{CONSIDERAÇÕES FINAIS}

As práticas de cuidado em saúde bucal precisam avançar na perspectiva de uma clínica ampliada baseada em tecnologias levesrelacionais, caracterizada pela escuta e produção de diálogo, vínculo e subjetividades, por meio do compartilhamento e reconstrução do plano terapêutico. Nessa clínica, o diálogo inicial e a identificação do motivo que trouxe a pessoa ao serviço de saúde devem ser realizados antes de se pensar qualquer estratégia que envolva o 'tratamento dentário' propriamente dito.

Para que essas novas possibilidades de abordagem clínica na odontologia possam estar presentes no cotidiano do cuidado, os currículos da graduação devem incluir bases teóricas e oportunidade de vivências e simulações clínicas com foco nas tecnologias leves-relacionais. O espaço dessas perspectivas teóricas na educação dos profissionais da saúde não se restringe à graduação, mas seguem como fundamentais na 
formação de pós-graduação, na formação de professores universitários e na educação permanente dos trabalhadores do SUS.

\section{ABSTRACT \\ Health care production focused on the Expanded Clinic: a necessary debate in Dental education}

This article discusses the health care production focused on Expanded Clinical Practice in Dental education. It is organized into four main themes of analysis that are the production of health care; humanization in health and expanded clinical practice; innovations in the production of care in oral health practices and Person-Centered Care. It considers that the health care based on relational technologies, characterized by listening and dialogue production, connecting and subjectivities through sharing and reconstruction of the therapeutic plan, must guide the formation of graduated dentists with more integral and humanized approach to oral health care and incorporate the guidelines of the Sistema Único de Saúde (SUS) (Brazilian Unified Health System) into its know-how. It presents the Person-Centered Care as one of the ways of acting in health care directed to the user and their potentialities, not to the disease. The undergraduate curricula of dentistry courses must include theoretical bases, opportunity of experiences and clinical simulations focusing on relational technologies. Such theoretical perspectives should not be restricted to undergraduate, but rather to be fundamental in the education of health professionals in postgraduate training, in the training of university professors and in the permanent education of SUS workers.

Descriptors: Dental Education. Oral Health. Comprehensive Health Care.

\section{REFERÊNCIAS}

1. Brasil. Ministério da Saúde. Portaria $\mathrm{n}^{\circ}$ 1.444/GM, de 28 de dezembro de 2000. Estabelece incentivo financeiro para a reorganização da atenção à saúde bucal prestada nos municípios por meio do
Programa de Saúde da Família. Diário Oficial [da] República Federativa do Brasil. 2000 dez.

2. Brasil. Ministério da Saúde. Secretaria de Atenção à Saúde. Departamento de Atenção Básica. Coordenação Nacional de Saúde Bucal. Diretrizes da Política Nacional de Saúde Bucal [Internet]. Brasília, 2004 [Acesso em 29 jul. 2015]. Disponível em: http://bvsms.saude.gov.br/bvs/publicacoes/ politica_nacional_brasil_sorridente.pdf.

3. Brasil. Conselho Nacional de Educação. Câmara de Educação Superior. Resolução CNE/CES 3/2002, de 19 de fevereiro de 2002. Institui as Diretrizes Curriculares Nacionais do Curso de Graduação em Odontologia. Diário Oficial [da] República Federativa do Brasil. 2002 mar. Seção 1. p. 10.

4. Brasil. Ministério da Saúde. Ministério da Educação. Programa nacional de reorientação da formação profissional em saúde - PRÓ-SAÚDE: objetivos, implementação e desenvolvimento potencial [Internet]. Brasília, 2007 [Acesso em 29 jul. 2015]. Disponível em: http://www.prosaude. org/rel/pro_saude1.pdf.

5. Brasil. Portaria Interministerial $n^{\circ} 421$, de 3 de março de 2010. Institui o Programa de Educação pelo Trabalho para a Saúde (PET Saúde) e dá outras providências. Diário Oficial da União. 2010 mar.

6. Brasil. Lei 11.129, de 30 de junho de 2005. Diário Oficial da União. 2005 jul.

7. Barros RS, Botazzo C. Subjetividade e clínica na atenção básica: narrativas, histórias de vida e realidade social. Ciênc Saúde Coletiva. 2011;16(11): 4337-48.

8. Ceron M. Habilidades de Comunicação: abordagem centrada na pessoa [Internet]. UNASUS, 2015 [Acesso em 5 jan. 2017]. Disponível em: www.unasus.unifesp.br/ 
biblioteca_virtual/esf/1/.../Unidade_17.pdf.

9. Merhy EE, Franco TB. Por uma composição técnica do trabalho centrada nas tecnologias leves e no campo relacional. Saúde Debate. 2003; 27(65):316-23.

10. Severino AJ. Metodologia do trabalho científico. 23 ed. São Paulo: Cortez; 2007.

11. Ayres JRCM. Cuidado e reconstrução das práticas de Saúde. Interface Comun Saúde Educ. 2004; 8(14): 73-92.

12. Junqueira MFPS. Cuidado: as fronteiras da integralidade. Ciênc Saúde Coletiva [Internet]. 2005; 10(3): 784-5.

13. Pinheiro R. Cuidado em Saúde. In: Pereira IB, Lima JCF organizadores. Dicionário da Educação Profissional em Saúde [Internet]. 2 ed. Rio de Janeiro: Fundação Oswaldo Cruz. Escola Politécnica de Saúde Joaquim Venâncio; 2009 [Acesso em 22 set. 2015]. Disponível em: http://www.sites.epsjv.fio cruz .br/dicionario/verbetes/cuisau.html.

14. Franco TB. As redes na micropolítica do processo de trabalho em saúde. In: Pinheiro R, Mattos R. organizadores. Gestão em Redes. Rio de Janeiro: CEPESCIMS/UERJLAPPIS; 2006.

15. Costa CB. Saúde bucal na Estratégia Saúde da Família: avanços e desafios na produção do cuidado odontológico [dissertação]. Florianópolis: Universidade Federal de Santa Catarina; 2015.

16. Merhy EE. Em busca do tempo perdido: a micropolítica do trabalho vivo em saúde. In: Merhy EE, Onocko R. organizadores. Agir em saúde: um desafio para o público. São Paulo: Hucitec; 1997. p. 71-112.

17. Mota LQ, Santos TA, Magalhães DBL. Humanização no Atendimento Odontológico: acolhimento da subjetividade dos pacientes atendidos por alunos de graduação nos campos de estágio. R Bras Ci Saúde. 2012; 16(4): 537-44.
18. Yujra VQ, Leite ET, Carvalho NM, Graff VA. Tecnologia Leve na Atenção em Saúde Bucal - cartografia do primeiro encontro clínico. In: Anais do $10^{\circ}$ Congresso Internacional da Rede Unida [Internet], 2012; Rio de Janeiro. Rio de Janeiro: Rede Unida; 2012 [Acesso em 30 jul. 2015]. Disponível em: http://conferencias.redeunida.org.br/ ocs/index.php/congresso2012/CRU10/paper /view/7484.

19. Barros RS, Wimmer GF, Botazzo C. Saúde Bucal e práxis no cotidiano dos serviços de saúde. BIS, Bol. Inst. Saúde (Impr.). 2007 abr; 41.

20. Merhy, EE. Em busca de ferramentas analisadoras das tecnologias em saúde: a informação e o dia a dia de um serviço, interrogando e gerindo trabalho em saúde. In: Merhy EE, Onocko R. Org. Agir em saúde: um desafio para o público. São Paulo: Hucitec; 1997. p. 113-50.

21. Cavaca AG, Gentilli V, Marcolino EM, Emmerich A. As representações da saúde bucal na mídia impressa. Interface Comun Saúde Educ. 2012 out/nov; 16(43):1055-68.

22. Brasil. Ministério da Saúde. Secretaria de Atenção à Saúde. Núcleo Técnico da Política Nacional de Humanização. Clínica ampliada, equipe de referência e projeto terapêutico singular. 2. ed. Brasília; 2008.

23. Santos AM. Organização das ações em saúde bucal na estratégia de saúde da família: ações individuais e coletivas baseadas em dispositivos relacionais e instituintes. Rev Atenção Prim Saúde. 2006; 9(2): 190-200.

24. Braid LMC, Machado MFAS, Aranha AC. Estado da arte das pesquisas sobre currículo em cursos de formação de profissionais da área da saúde: um levantamento a partir de artigos publicados entre 2005 e 2011. Interface Comun Saúde Educ. 2012 jul/set; 16(42): 679-92. 
25. Mitre SM, Siqueira-Batista R, Girardi-deMendonça JM, Morais-Pinto NM, Meirelles $\mathrm{CAB}$, Pinto-Porto $\mathrm{C}$, et al. Metodologias ativas de ensino-aprendizagem na formação profissional em saúde: debates atuais. Cienc Saúde Colet. 2008; 13(2):2133-44.

26. Brasil. Ministério da Saúde. HumanizaSUS: Política Nacional de Humanização - a humanização como eixo norteador das práticas de atenção e gestão em todas as instâncias do SUS [Internet]. Brasília, 2004 [Acesso em 28 jul. 2015]. Disponível em: http://bvsms.saude.gov.br/bvs/publicacoes/ humanizasus_2004.pdf.

27. Hayacibara MF, Lotti LF, Terada RSS, Hidalgo MM, Bispo CGC, Terada HH, et al. Experiência de Clínica Ampliada em Odontologia na Universidade Estadual de Maringá. Rev Bras Educ Méd. 2012:36(1) (supl 2):178-83.

28. Pereira EHP, Barros RDB. Humanização. In: Pereira IB, Lima JCF organizadores. Dicionário da Educação Profissional em Saúde [Internet]. Rio de Janeiro: Fundação Oswaldo Cruz. Escola Politécnica de Saúde Joaquim Venâncio. 2. ed. 2009 [Acesso em 1 out. 2015]. Disponível em: http://www.sites.epsjv.fiocruz.br/dicionario /verbetes/hum.html.

29. Vasconcelos EM, Fratucci MVB. Práticas de saúde bucal [Internet]. UNASUS, 2015 [Acesso em 10 jan. 2017]. Disponível em: http://www.unasus.unifesp.br/biblioteca_vir tual/esf/2/unidades_conteudos/unidade1 5o/unidade15o.pdf.

30. Cunha GT. A construção da clínica ampliada na atenção básica. 3. ed. São Paulo: Hucitec; 2010.

31. Campos GWS. Saúde Paidéia. São Paulo: Hucitec; 2003.

32. Brasil. Ministério da Saúde. Secretaria de Atenção à Saúde. Núcleo Técnico da Política
Nacional de Humanização. Clínica ampliada e compartilhada. Brasília; 2009.

33. Godoy DC. O ensino da clínica ampliada na atenção primária à saúde: percepções e vivências de alunos de graduação médica [dissertação]. Botucatu: Universidade Estadual Paulista Júlio de Mesquita Filho; 2013.

34. Campos GWS, Amaral MA. A clínica ampliada e compartilhada, a gestão democrática e redes de atenção como referenciais teórico-operacionais para a reforma do hospital. Ciênc Saúde Coletiva. 2007; 12(4):849-59.

35. Botazzo C, Souza CR, Zilbovicius C, Cunha CSGF, Pires FS, Carrer FCA, et al. Inovação na produção do cuidado em saúde bucal. Possibilidades de uma nova abordagem na clínica odontológica para o Sistema Único de Saúde. Relatório Técnico. São Paulo; 2015.

36. 36. Pires FS, Botazzo C. Organização tecnológica do trabalho em saúde bucal no SUS: uma arqueologia da política nacional de saúde bucal. Saúde Soc.; 24(1): 273-84.

37. Souza CR, Botazzo C, Clara AS, Pires FS, Fonsêca GS, Takita SMY, et al. Anamnese coletiva e consulta individual como instrumentos de ampliação do cuidado em saúde bucal. In: Anais do $11^{\circ}$ Congresso Brasileiro de Saúde Coletiva [Internet], 2015; Goiânia. Goiânia: ABRASCO; 2015.

38. Bacellar A, Rocha JSX, Flôr MS. Abordagem centrada na pessoa e políticas públicas de saúde brasileiras do século XXI: uma aproximação possível. Rev NUFEN. 2012;4(1):127-40.

39. Gobbi SL, Missel ST. Vocabulário e noções básicas da Abordagem Centrada na Pessoa. 2. ed. São Paulo: Vetor; 2002.

40. Mills I, Frost J, Cooper C, Moles DR, Kay E. Patient-centred care in general dental 
practice: a systematic review of the literature. Correspondência para:

BMC Oral Health. 2014;14(64):1-13. Ramona Fernanda Ceriotti Toassi

41. Mills I, Frost J, Kay E, Moles DR. Person- e-mail: ramona.fernanda@ufrgs.br centred care in dentistry the patients' Faculdade de Odontologia da UFRGS

perspective. Br Dent J. 2015; 218(7):407-13. Rua Ramiro Barcelos 2492, 2o andar 90035-003 Porto Alegre, RS 